\title{
AVANCES EN LA INVESTIGACIÓN: LA ENERGÍA COMO SISTEMA COMPLEJO EN EL PROYECTO DEL HÁBITAT. UNA MIRADA DESDE LA ENSEÑANZA
}

\author{
Eje 4: Hábitat e infraestructura
}

\author{
Esp. Arq. Bellot Rodolfo \\ Dr. Arq. Diego Fiscarelli
}

\begin{abstract}
Trabajo de Investigación de la tesis doctoral en el marco del Doctorado en Arquitectura por ante la Facultad de Arquitectura, Diseño y Urbanismo. Universidad Nacional del Litoral, Santa Fe, Argentina, rjbellot@fadu.unl.edu.ar
\end{abstract}

\section{RESUMEN}

Existe un inquietante interés por parte de los alumnos de arquitectura, de profesionales, de organismos gubernamentales y no gubernamentales, de instituciones y medio social en relación al uso racional y mejor aprovechamiento de la energía. Concretamente en esta actividad basta con ver los avances de los Estados -municipales, provinciales y nacional- en materia de sustentabilidad, eficiencia y etiquetado energético entre otras para tener una muestra de este fuerte requerimiento relacionado con la producción y uso edilicio. Considerando esto que se denominará demanda social (DS) y el creciente interés en el tema por parte de la academia (IA), es que es oportuno el momento para estudiar y reflexionar acerca de la enseñanza de la arquitectura bajo esta perspectiva.

Este trabajo tiene como intención contar objetivos y avances de una tesis doctoral llevada adelante en la FADU-UNL, denominada "La energía como sistema complejo en el proyecto del hábitat. Una mirada desde la enseñanza", que a su vez tiene como propósito identificar desde el campo disciplinar de la enseñanza de la arquitectura, el cuerpo de conocimientos relacionados al tema energético que fortalezcan la formación de profesionales comprometidos con los procesos productivos, especialmente los regionales, involucrados con la equidad social en relación a una reducción de consumo energético, una mejora en la calidad ambiental y que a la vez mitiguen los impactos del hábitat construido.

Una vez analizada la representación de la energía como un sistema complejo se podrá inferir cuáles de todas sus aristas son significativas para la formación de profesionales con competencias para proyectar y construir espacios destinados al hábitat.

Con respecto al estado de la educación en la formación profesional del arquitecto, tema que se tratará con mayores detalles al igual que el resto de los conceptos que se están desgranando en el intento de explicarlos enunciativamente, se puede inferir que la clásica división en tres áreas de conocimientos, "Diseño-Tecnología-Ciencias Sociales" como unidades epistemológicas que configuran los distintos sectores del saber de la carrera, en un intento de control total no hizo más que fragmentar sus espacios como claustros más o menos rígidos. El problema energético y todos 
sus derivados en relación a la sostenibilidad, la eficiencia, el aprovechamiento de los medios naturales y el cuidado ambiental pueden darnos una oportunidad inmejorable para renovar el dialogo que parecía haber muerto hace mucho tiempo entre las diferentes disciplinas de las diferentes áreas académicas del conocimiento

\section{PALABRAS CLAVE: ENERGÍA - ENSEÑANZA DE LA ARQUITECTURA - SISTEMA - HÁBITAT}

\section{FUNDAMENTACIÓN}

Todos los actores vinculados con la disciplina arquitectónica podemos observar las derivaciones del cambio climático, la disminución de hielos, el aumento de la temperatura promedio, los cambios de intensidad, duración y frecuencia de lluvias, etc.; algunas de estas consecuencias se las puede verificar en las conclusiones que anualmente difunde la Convención Marco de las Naciones Unidas sobre el Cambio Climático (UNFCCC, 2018). En general se puede afirmar que los orígenes del cambio climático se manifiestan en las emisiones por exceso de gases de efecto invernadero (GEI), principalmente $\mathrm{CO} 2$, a la atmósfera, parte de esas emisiones, aproximadamente un $40 \%$, se relacionan con la industria de la construcción, "producción" y el funcionamiento de obra de arquitectura, "uso". (International Energy Agency, 2017).

Resulta importante -y hasta necesario- pensar qué respuesta se puede dar o qué aporte se consigue realizar desde lo que hoy se denomina "arquitectura sustentable" y los conceptos teóricos que ese término implica, para contribuir a la disminución de las emisiones.

Teniendo en cuenta lo expresado, podríamos preguntar: ¿Se puede pensar en una arquitectura de bajas emisiones? ¿Es posible desarrollar un tipo de arquitectura con bajo consumo de energía? ¿Se tiene la base de conocimientos para el abordaje del tema y así dar respuestas al problema?

El propósito de la Tesis Doctoral que da marco al presente trabajo es identificar, desde el campo disciplinar de la enseñanza de la arquitectura, el cuerpo de conocimientos relacionados al tema "energía" que fortalezcan la formación de profesionales comprometidos con los procesos productivos, especialmente los regionales, comprometidos con la equidad social en relación a una reducción de consumo energético, una mejora en la calidad ambiental y que a la vez mitiguen los impactos sobre el hábitat construido.

En primer lugar se debe entender y definir qué es energía y energía como un sistema complejo ${ }^{1}$, para luego plantear y problematizar la cosa en función de la demanda de la sociedad y el medio, del estado general de la enseñanza de arquitectura -en la formación de grado- y en virtud de una sospechada demanda e interés académico por el tema. ${ }^{2}$

Al mismo tiempo cuando se hace referencia al término, se acuñan conceptos mucho más amplios en los que es necesario realizar un recorte para acotarlo al objeto de estudio.

\footnotetext{
${ }^{1}$ Sistema como entidad con límites y con partes interrelacionadas, en la cual su suma es exponencial respecto a la suma de las partes. Complejo según Edgar Morín: tejido de constituyentes heterogéneos inseparablemente asociados. (ver pensamiento complejo)

${ }^{2}$ Se plantearán estos tres ejes de discusión como planteamiento del problema, ver cuadro $n^{\circ} 2$.
} 
El vocablo siempre estuvo presente en la arquitectura, pero ahora (re) significado3, y lo está en los cuatro estadios de la arquitectura, como inversión de recursos, como expresión cultural, como filtro ambiental y como contenedor de actividades (Broadbent, 1976)

Si se propusiera un ejercicio didáctico y lúdico al mismo tiempo, en donde el juego consistiría en generar una lluvia de ideas conceptuales con términos que se asocien a energía en la arquitectura, se podría obtener el siguiente esquema: (cuadro $n^{\circ} 1$ ).

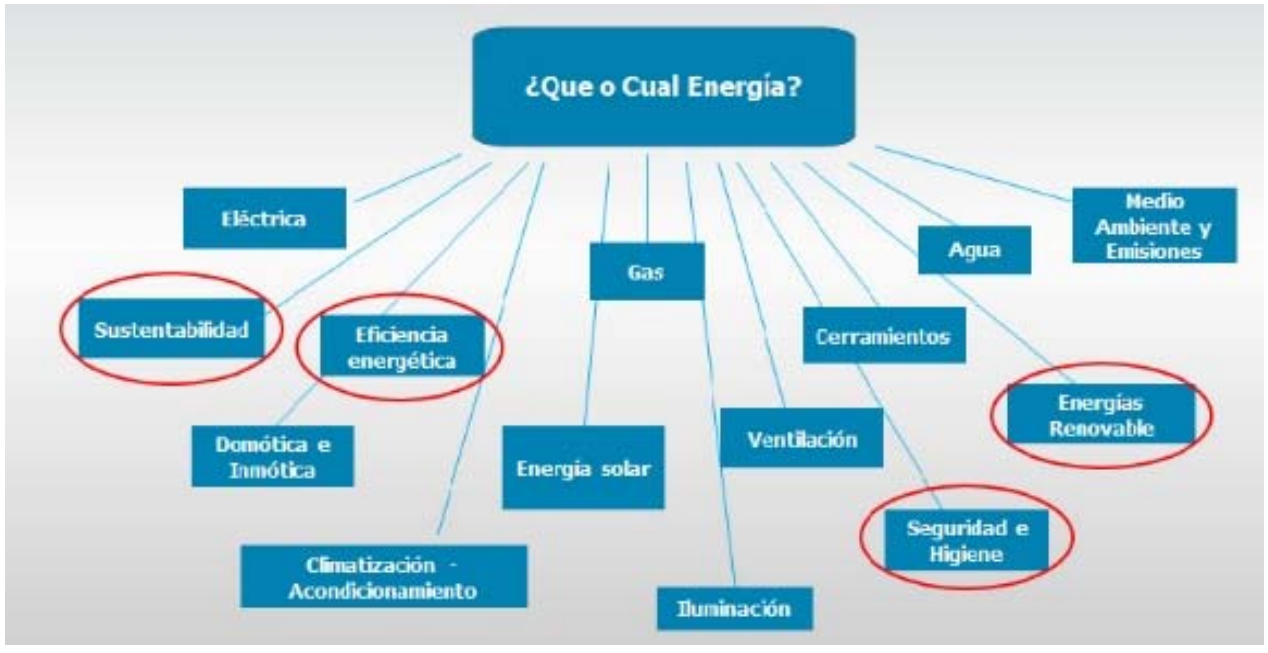

Cuadro $\mathrm{N}^{\circ} 1$ de elaboración propia

Ahora bien, de todos estos términos, de los que revisten interés e importancia se puede enunciar:

Eficiencia energética: es una práctica y a su vez manera de gestionar el incremento de la energía, obteniendo un resultado igual con menor consumo o un resultado mayor consumiendo lo mismo. (International Energy Agency, 2017). Es un mecanismo por el cual se procura que todos los procesos y productos optimicen el consumo de energía para de esta forma reducir el gasto, bajar costos y colaborar con el medio, ya que se liberan menos GEI. Dentro de este concepto se encuentra el de etiquetado energético de artefactos y de inmuebles, el uso racional de la energía y la educación energética.

Energía renovable: se denominan así a todas aquellas energías que se producen naturalmente y de forma inagotable, contando su producción con la mejor relación-equilibrio con el planeta. Entre las energías más comunes que se pueden generar bajo el concepto de renovables se encuentran la solar tanto fotovoltaica como térmica, hidráulica, eólica, biomasa, etc. Las energías renovables tienen los menores índices de emisiones de GEI por lo tanto se las llama también energías limpias o energías naturales.

Arquitectura sustentable: se entiende por este concepto un modo de vincular el diseño con las tecnologías de forma tal que se aprovechen al máximo los recursos naturales reduciendo así el consumo artificial o no natural sin por ello bajar los niveles de confort. Es un equilibrio entre sistemas pasivos, sistemas activos y sistemas mixtos. Incluye el ciclo de vida de los materiales, el tratamiento de residuos, la gestión de la energía y la arquitectura bioclimática entre otros.

\footnotetext{
${ }^{3}$ Significación del termino energía, por el Dr. Arq. Raul Ajmat en el seminario "El rol de la energía en la concepción y diseño del hábitat construido", en el marco del Doctorado en Arquitectura FADU-UNL (2015).
} 
Seguridad e higiene: es necesario despojar en este término el concepto de seguridad e higiene laboral, para considerarlo desde la vinculación con la energía mediante la higiene ambiental propia de la contaminación y tratamiento de efluentes, se procura un ambiente con la menor cantidad de partículas nocivas, con la menor emisión de GEI. Se asocian a este concepto los procesos de producción seguros y menos perjudiciales para el medio.

Estos términos-conceptos además de considerarse transversales unos con otros, se los puede asumir como abarcativos de los demás mencionados: electricidad, gas, agua, ventilaciones, iluminación, domótica, Inmótica, cerramientos, acondicionamiento, residuos y efluentes, balances térmicos, etc.

Con respecto al estado de la educación en la formación profesional del arquitecto se puede inferir que la clásica división en tres áreas de conocimientos, "Diseño-Tecnología-Ciencias Sociales" como unidades epistemológicas que configuran los distintos sectores del saber de la carrera, en un intento de control total no hizo más que fragmentar sus espacios como claustros más o menos rígidos. El problema energético y todos sus derivados en relación a la sostenibilidad, la eficiencia, el aprovechamiento de los medios naturales y el cuidado ambiental pueden darnos una oportunidad inmejorable para renovar el dialogo que parecía haber muerto hace mucho tiempo entre las diferentes disciplinas de las diferentes áreas académicas del conocimiento.

\section{PROBLEMA DE INVESTIGACIÓN}

En relación a la enseñanza, se abordará desde tres ejes que configuran, modelan y problematizan al tema de la energía, (cuadro $n^{\circ} 2$ ).

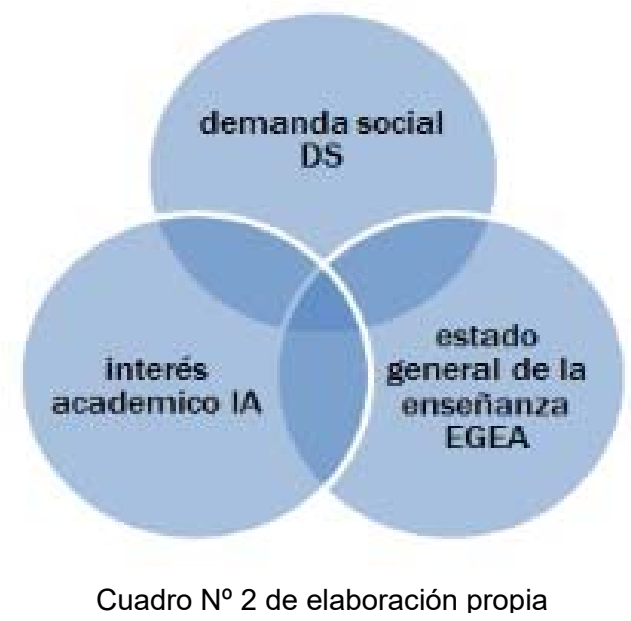

Cuando se habla de Demanda Social, en adelante "DS", se hace clara referencia a un pedido, requerimiento o necesidad que instala una presión en dirección a un cambio en algunas de las relaciones sociales establecidas y constituidas. Al respecto si se consideran los problemas energéticos y ambientales ut supra detallados, el aumento del nivel del mar, la gran emisión de gases de efecto invernadero, la perdida de bosques y selvas, el calentamiento del planeta, la escases de recursos naturales y el agotamiento de los combustible no renovables, se puede ver con claridad que existe un consenso para revertir estos problemas, un consenso en donde no está claro si definitivamente se está en acción o aun es un discurso correcto que no pasa más allá de una expresión de deseo. "La prueba es que recibimos todas estas noticias con una calma asombrosa, e incluso con un tipo de estoicismo admirable" (Latour, 2017, pág. 22). No obstante, 
en el consenso, existen numerosos intentos, tal vez débiles, que procuran revertir el nuevo régimen.

Cuando se habla de Interés Académico "IA" es notable y muy interesante observar el creciente entusiasmo en estudiantes y jóvenes graduados por conocer e investigar en temas relacionados con la problemática planteada. Para el abordaje de esta línea, definitivamente importante, es necesario que se construya una red que se ira delineando entre las agencias ${ }^{4}$ que conforman la realidad que se intenta describir.

Habiéndose llegado a este punto, es interés analizar el estado general de la enseñanza de la arquitectura EGEA en relación a temas vinculados con lo energético, para ello se considerara, en principio, los contenidos, incumbencias y actividades reservadas al título de arquitecto

Para esto se deberán analizar las normativas vigentes en referencia a Leyes de Educación Superior, incumbencias profesionales y actividades reservadas en la arquitectura, e incluso plantear un recorte a partir de la propia currícula y los planos de estudio de las casa de formación.

Existe un reciente documento académico: "Lineamientos para la mejora de la enseñanza sobre eficiencia energética en carreras estratégicas de ingeniería y arquitectura”, editado en el año 2017, el mismo trata sobre la situación actual respecto de la competencias necesarias relacionadas a temas energéticos y las estrategias de acción para implementar en la enseñanza, al respecto se clasificaron por consenso, importancia y mención las competencias como necesarias en la formación de los futuros arquitectos e ingenieros. Se muestran y resaltan las más relevantes, (cuadro $n^{\circ} 3$ ). También se indica la clasificación por categorías, las mismas fueron determinadas en tres estadios: competencias transversales de formación, competencias éticoprofesionales y competencias de formación técnico-profesional, (cuadro $n^{\circ} 4$ ).

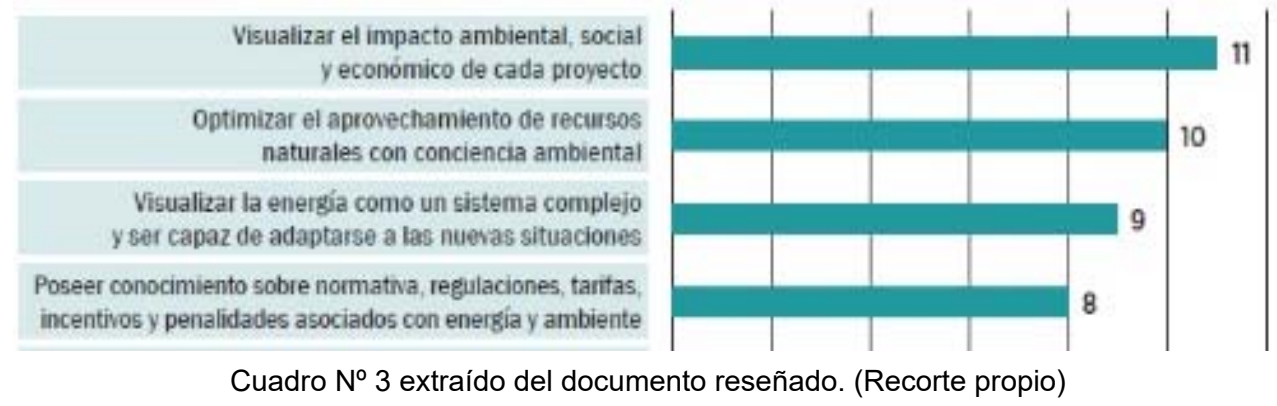

\footnotetext{
${ }^{4}$ Agency/ies es el concepto de Latour al referirse a los actantes objetos y sujetos.
} 


\begin{tabular}{|c|c|c|c|}
\hline $\begin{array}{l}\text { COMPETENCIAS DE FORMACIÓN } \\
\text { (a desarrollar de acuerdo con el perfil de egresado) }\end{array}$ & Transversal & $\begin{array}{l}\text { Ético- } \\
\text { Profesional }\end{array}$ & $\begin{array}{l}\text { Técnico- } \\
\text { Profesional }\end{array}$ \\
\hline Analizar criticamente la matriz energética nacional & $\mathrm{x}$ & & $\mathrm{x}$ \\
\hline $\begin{array}{l}\text { Visualizar la energia como un sistema complejo y ser capaz } \\
\text { de adaptarse a las nuevas situaciones }\end{array}$ & $\mathrm{x}$ & $\mathrm{x}$ & $\mathrm{x}$ \\
\hline Interactuar en situaciones prácticas multiculturales & $\mathrm{x}$ & $x$ & \\
\hline Comunicar y dar visibilidad a la problemática & & $\mathrm{x}$ & $\mathrm{x}$ \\
\hline Trabajar en equipos interdisciplinarios & $x$ & $\mathrm{x}$ & \\
\hline $\begin{array}{l}\text { Operar con herramientas informáticas tales como software } \\
\text { de simulación, entre otros }\end{array}$ & $\mathrm{x}$ & & $\mathrm{x}$ \\
\hline Visualizar el impacto ambiental, social y económico de cada proyecto & $\mathrm{x}$ & $\mathrm{x}$ & $\mathrm{x}$ \\
\hline $\begin{array}{l}\text { Evaluar la eficiencia energética en la optimización de procesos } \\
\text { específicos }\end{array}$ & & & $\mathrm{x}$ \\
\hline $\begin{array}{l}\text { Optimizar el aprovechamiento de recursos naturales con } \\
\text { conciencia ambiental }\end{array}$ & $\mathrm{x}$ & $\mathrm{x}$ & $\mathrm{x}$ \\
\hline $\begin{array}{l}\text { Evaluar el beneficio técnico-económico de la Eficiencia Energética } \\
\text { de manera sostenible }\end{array}$ & & $\mathrm{x}$ & $x$ \\
\hline $\begin{array}{l}\text { Analizar procesos y ciclo de vida de cada producto en el contexto } \\
\text { de la demanda de energia }\end{array}$ & & & $\mathrm{x}$ \\
\hline Formar grupos con foco en el ahorro energético & $\mathrm{x}$ & $\mathrm{x}$ & \\
\hline $\begin{array}{l}\text { Poseer conocimiento sobre las leyes, regulaciones, normativas, tari- } \\
\text { fas, incentivos y penalidades asociados con la energia y el ambiente }\end{array}$ & $\mathrm{x}$ & & $\mathrm{x}$ \\
\hline Diseñar, valorar y realizar prototipos de acuerdo al impacto & & & $\mathrm{x}$ \\
\hline
\end{tabular}

Cuadro $N^{\circ} 4$ extraído del documento reseñado.

\section{MARCO TEÓRICO}

En relación con la construcción del marco conceptual, el enfoque del trabajo que se presenta reconoce al constructivismo como la corriente de pensamiento que dará sustento a los objetivos e hipótesis. Con respecto al término "constructivismo", es el nombre que se asignó a varias corrientes que nacieron en el seno de las artes en general, literatura, pintura y escultura, en la filosofía, la arquitectura, la pedagogía y en las ciencias sociales en general.

De esas muchas aristas y posturas, encontramos dentro de la perspectiva antropológica que existe una mirada teórica donde el sujeto (hombre) es incompleto, inacabado y debe construirse, está capacitado para elaborar su realidad y su propio conocimiento de la realidad; y desde una perspectiva epistemológica se encuentra la ruptura con el programa moderno. Empero hay dos posiciones del constructivismo que son muy interesantes para esta ponencia, la educativa y la filosófica, la primera se abordará desde un breve repaso de orden general, la segunda desde un pensador actual como referente de la misma.

Dentro del constructivismo educativo o en la educación, son varias las corrientes que han surgido y las teorías planteadas, se hará hincapié en las más generalizadas. Muy por el contrario de las teorías asociacionistas del aprendizaje, aquellas que epistemológicamente se unifican con el conductismo, están las teorías cognitivas, epistemológicamente constructivas.

A la primera corriente algunos la denominan desarrollista, evolucionista o teoría de la equilibración, mira al sujeto (hombre) como un ser activo que interactuando con otros y con el medio desarrolla sus capacidades y conocimientos. En esta corriente los contenidos sólo son un medio que permite pensar y deducir. Jean Piaget es quien embandera esta teoría, el aprendizaje tiene su origen en la acción. Los estudiantes adquieren los conocimientos mediante su propia acción y el docente es quien crea el clima. "El aprendizaje es un proceso mediante el cual el sujeto, a través de la experiencia, la manipulación de objetos, la interacción con las personas 
genera o construye conocimiento, modificando, en forma activa sus esquemas cognoscitivos del mundo que lo rodea, mediante el proceso de asimilación" (Díaz, Manterota, 2013: 74).

La corriente del aprendizaje social, identificada con Lev Vygotsky (1896-1934), plantea que el aprendizaje se desarrolla siempre en un contexto compartido, en grupos, con un colectivo de trabajo. Se rechaza toda labor que reduce el aprendizaje a simples asociaciones entre estímulos y respuestas, el conocimiento se construye por medio de operaciones y habilidades cognoscitivas que se inducen en la interacción social. Para este filósofo el desarrollo intelectual no es independiente del medio social. "Vygotsky esboza el concepto de zonas de desarrollo próximo, la distancia entre el nivel real de desarrollo determinado por la capacidad de resolver un problema o una situación y desarrollo potencial, determinado a través de la resolución de un problema bajo la guía de un compañero más capaz". (Baquero, 1997)

Otra escuela es la que pone énfasis en los contenidos, "Teoría del aprendizaje significativo por descubrimiento", tiene a David Ausubel (1918-2008) como referente, sostiene que el aprender ocurre si se relaciona de forma sustancial y no arbitraria. Toda la información nueva junto a los conocimientos previos que el sujeto posee son, en su estructura cognitiva, hilvanados a la disposición de aprender significativamente. El aprendizaje es significativo siempre y cuando los contenidos se relacionen de manera no memorística, con motivación y actitud, la nueva información se conecta con algún concepto relevante que ya fue adquirido, y funciona como un anclaje. Existen tres tipos de aprendizaje significativo, según el objeto aprendido: representacional, de conceptos y proposicional (Rodríguez Palmero, Moreira, Caballero Sahelices, \& Greca, 2008).

La corriente de las habilidades cognoscitivas con la teoría de las inteligencias múltiples de Howard Gardner (1943), afirma que la inteligencia es la capacidad para resolver problemas o para elaborar productos y respuestas que sean valiosos. La facilidad que tengamos para aprender algo y cómo lo hacemos dependerá del tipo de inteligencia que predomine en el individuo. Desarrolla en su teoría ocho inteligencia: lógica, matemática, lingüística, espacial, musical, corporal-kinestésica, intrapersonal y naturalista. (Gardner, 2015).

En cuanto al constructivismo filosófico, es intención observarlo desde el constructivismo de Bruno Latour, es intención abordar a un autor actual y referente, a la hora de hablar del tema. Además de presentarnos un aporte doble ya que investiga desde hace muchos años la relación de las ciencias para con el cambio climático y el nuevo régimen ambiental. Es reconocido por sus aportes a la antropología de las ciencias con su teoría del Actor-Red y creador, dentro del área de investigación, del innovador médialab, el cual investiga sobre filosofías del ambiente, controversias climáticas desde las conexiones sociales, técnicas y económicas, entre otras.

Latour no coincide con la perspectiva realista, ya que no da como real a la objetividad de los hechos, pero tampoco está de acuerdo con la sociología del conocimiento de lo que se conoce como programa fuerte, esto es básicamente que la ciencia sólo se puede analizar desde categorías sociológicas.

Si bien no es naturalista (realista), ni deconstruccionista (relativista), Latour (2003) resume sus ideas cuando dice;

"Lejos de intentar explicar los sólidos hechos científicos a través de los endebles hechos de las ciencias sociales, el objetivo ha llegado a ser el de entender cómo la ciencia y la tecnología han 
proporcionado algunos de los ingredientes necesarios para dar cuenta de la formación y la estabilidad de la sociedad misma. Este es el único modo de conceder algo de su significado original al término 'construcción', de hacer hincapié en ese proceso colectivo que da lugar a una construcción sólida a través de la movilización de oficios, componentes y coordinaciones de diverso orden." (Citado por Loredo Narciandi, 2009: 117).

En sus escritos plasma una permanente crítica a la modernidad, especialmente a sus dualismos (sujeto-objeto, naturaleza-cultura, ciencia-religión), y los expresa como algo superado, no se detiene demasiado a reinterpretarlos o redefinirlos

En su último libro, "Cara a cara con el planeta. Una nueva mirada sobre el cambio climático, alejada de las posiciones apocalípticas" Bruno Latour logra un ensayo que permite sintetizar sus investigaciones ya conocidas en obras como, "Nunca fuimos modernos", "Reensamblar lo social", "La esperanza de pandora", entre otros. En este libro puede verse el punto de vista que, según Latour, los modernos 5 tienen con relación a la naturaleza en torno a lo que él denomina el nuevo régimen climático.

Propone politizar la ecología, esto significa construir acciones desde una postura crítica y reflexiva como terrestres, sin miedo a exclamar el dilema existencial en el que se está.

En el juego de representaciones que se expone en la última conferencia, interpela claramente a la comunidad científica, en donde le pide básicamente que ante los conflictos ecológicos, representen cabalmente sus objetos de estudio, sus posturas e intereses, para negociar punto por punto hasta descubrir las posibilidades de actuar. Es un llamado a politizar la ciencia como recurso para enfrentar la crisis ecológica.

Ahora bien, ¿por qué constructivismo para un marco teórico? En este pequeño viaje que se intentó realizar por lo que se denomina "Constructivismo", se abre un enorme panorama de las distintas posturas. Se remarca el término "distintas" desde un punto de vista epistemológico en relación a la teoría constructivista del sujeto. El constructivismo educativo de Piaget, al que se acusa de formalismo (se deben recordar los móviles inmutables, esas estructuras fijas ligadas al sujeto) se contrapone con el constructivismo de Latour que cae de lleno en un estructuralismo (esa estructura trama compleja que hace acontecer al mundo). Uno le brinda al sujeto la capacidad de agencialidad6 mientras que el otro la extirpa.

Entonces, en este punto, sería prudente volver a recordar la definición inicial, aquella que establecía algunos puntos de contacto que permitían definir la corriente que se estudia, toda acción trae aparejada consecuencias y por ende es previa a los hechos, el conocimiento llega a los agentes que han sido capaces de construirse, el lenguaje se debe reemplazar por uno formal y específico, se rechaza la existencia de una realidad ya que ésta es una construcción (creación) de quien la observa a partir de sus percepciones y datos previos, se rechaza el fetichismo por los objetos, etc.

Con respecto a Bruno Latour, dada su contemporaneidad y particular modo de entender la manera de pensar la ciencia y en el que puede verse un hilo conductor, tal cual se analizara de su último libro que responde a lineamientos de investigación de más de cuatro décadas, no menos cierto es

\footnotetext{
${ }^{5}$ En el concepto de moderno Latour hace clara referencia a la cultura occidental, el periodo que surge desde las angustias del Renacimiento y que se prolonga hasta principios del siglo XX.

${ }^{6}$ Agencialidad (Agency/ies) concepto comentado en página 4 del presente documento.
} 
que ha tenido dos momentos o al menos son dos etapas que se pueden resaltar en su obra. Una de oposición total a la posturas realistas y racionalistas con un constructivismo radical y otra definida, por el mismo autor, en su texto "Las promesas del constructivismo" (2003), del cual podemos extraer una crítica al constructivismo que al mismo tiempo intenta rescatar, con un profundo intento de revisionismo, resignificando y corriendo conceptos como el de construccionismo social.

El autor plantea una solución composicionista, se diferencia claramente de la posición naturalista (para él superada y propia de la modernidad), algo muy interesante el concepto de composicionista ya que intenta juntar, pero sin perder heterogeneidad. Aquí radica una gran diferencia epistemológica, no se trata sólo de lo que está y lo que no está construido, sino de lo que está bien y lo que está mal construido. En la composición hay que debatir todos los puntos, exponer la ciencia, politizarla.

Se entiende que en esta corriente se pueden hallar los hilos conductores y los lentes en los que se pueden mirar los problemas de estudio. La enseñanza sin lugar a dudas necesita cada vez más del elaborado proceso de construcción, en donde alumnos, docentes, contenidos, currícula, normativa y tecnologías den respuesta a problemas actuales, con transferencia al medio y respetando tanto las futuras como las actuales generaciones.

\section{HIPOTESIS}

Las limitadas estrategias pedagógicas orientada a la comprensión de la energía como sistema complejo, en el marco de la formación universitaria en arquitectura, podrían representar obstáculos en el desarrollo de las competencias profesionales necesarias para abordar, desde la disciplina, las condicionantes del nuevo régimen climático.

\section{OBJETIVOS | OBJETIVOS GENERALES Y ESPECÍFICOS:}

- $\quad$ Contribuir, desde el campo disciplinar de la arquitectura, a los procesos de enseñanzaaprendizaje que permitan fortalezcan la formación de profesionales comprometidos con los procesos productivos, especialmente los regionales, comprometidos con la equidad social en relación a una reducción de consumo energético, una mejora en la calidad ambiental y que a la vez mitiguen los impactos del hábitat construido.

- Estimar sistemas pedagógicos innovadores o ciertamente efectivos que promuevan una mejora en relación a la enseñanza de la arquitectura.

- Fundamentar sistemas, herramientas y tecnologías orientadas al manejo y gestión energética edilicia, desde su etapa proyectual para favorecer el proceso de capacitación y enseñanza en la carrera de arquitectura

\section{CONCLUSIONES}

Lógicamente y como es de esperar en trabajos de investigación que están iniciándose, las conclusiones no son posibles desde la perspectiva de una verificación de objetivos e hipótesis, pero sí se puede esbozar una por los distintos estadios de corrección y evaluación que sumados a los seminarios específicos despierten en el tema una profunda expectativa e interés, especialmente desde la mirada docente, en donde el tema energía, tal cual se propone, es una temática relativamente nueva en la enseñanza superior y solamente se trata desde el empeño de algunas cátedras y no desde una inclusión en la currícula y planes de estudio, menos aún desde 
la construcción integral y compleja de las distintas áreas que conforman los núcleos epistémicos de la enseñanza de la arquitectura.

Por tal motivo se espera, luego de un profundo diagnóstico, poder presentar nuevos avances más promisorios en próximos encuentros en el Congreso Regional de Tecnología de Arquitectura.

\section{BIBLIOGRAFÌA}

Baquero, Ricardo (1997). Vigotsky y el aprendizaje escolar ( $2^{\circ}$ ed.). Buenos Aires: Aique Grupo Editor SA.

Broadbent, Geoffrey (1976). Diseño arquitectonico. Arquitectura y ciencias humanas. ( $2^{\circ}$ ed.). Barcelona: G.G.

Chevllard, Yves (2005). La transposición didactica. Del saber sabio al saber enseñado. Aique.

de Alba, Alicia (1998). Curriculum: crisis, mito y perspectivas. Buenos Aires: Miño y Davila.

de Garrido, Luis (2014). Arquitectura. Energía - Cero (1ºd.). Barcelona: Monsa.

FADU UNL. (2016). Encuesta graduados recientes. Santa Fe.

International Energy Agency (2017). iag.org. (IEA Publications, Ed.) Recuperado en febrero de 2018, de www.iag.org: https://www.iea.org

Latour, Bruno (2001). La esperanza de Pandora: Ensayos sobre la realidad de los estudios de la ciencia ( ${ }^{\circ}$ ed.). Barcelona: Gedisa.

Latour, Bruno (2017). Cara a cara con el planeta. Una nueva mirada sobre el cambio climático alejada de las posiciones apocalípticas. ( $1^{\circ} \mathrm{ed}$.). (D. Ariel, Trad.) Buenos Aires, Argentina: Siglo Veintiuno Editores.

Loredo Narciandi, José Carlos (enero-abril de 2009). ¿Sujetos o "actantes? El constructivismo de Latour y la psicología constructivista. Revista de Antropología Iberoamericana, 4(1), 113-136.

Ministerio de Energía y Minería \& Ministerio de Educación. (2017). Lineamientos para la mejora de la enseñanza sobre eficiencia energética en carreras estrategicas de ingeniería y arquitectura. Buenos Aires: Presidencia de la Nación.

Ministerio de Energía y Minería. (2015). argentina.gob.ar. Recuperado el 15 de febrero de 2018, de https://www.argentina.gob.ar

Morin, Edgar (2005). Introducción al pensamiento complejo. Barcelona: Gedisa.

Reyes, Roman (2009). Diccionario Crítico de Ciencias Sociales. Terminología Científico-Social. Constructivismo. (U. C. Madrid, Ed.) Madrid.

Rodríguez Palmero, Maria Luz, Moreira, Marco Antonio, Caballero Sahelices, Maria Concesa \& Greca, lleana Maria (2008). La teoria del aprendizaje significativo en la perspectiva de la psicología cognitiva ( $1^{\circ}$ ed.). Barcelona: Octaedro.

UNFCCC. (2018). unfccc.int/es. Obtenido de http://unfccc.int 